\title{
Lubiprostone improves visualization of small bowel for capsule endoscopy: a double-blind, placebo-controlled 2-way crossover study
}

\section{(ㄷ)(1) $\odot(9$}

\author{
Authors \\ Institutions \\ 1 Department of Gastroenterology and Hepatology, \\ Yokohama City University School of Medicine \\ 2 Office of Postgraduate Medical Education, Yokohama \\ City University Hospital \\ 3 Department of General Medicine, Yokohama City \\ University School of Medicine
}

Mizue Matsuura ${ }^{1}$, Masahiko Inamori ${ }^{2}$, Yumi Inou' ${ }^{1}$, Kenji Kanoshima ${ }^{1}$, Takuma Higurashi ${ }^{1}$, Hidenori Ohkubo ${ }^{1}$, Hiroshi lida', Hiroki Endo', Takashi Nonaka', Akihiko Kusakabe ${ }^{3}$, Shin Maeda², Atsushi Nakajima'

submitted 30.5.2016

accepted after revision 6.2 .2017

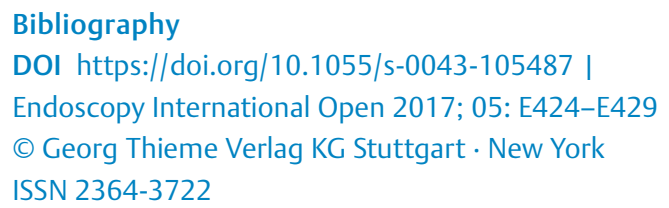

Corresponding author

Masahiko Inamori, M.D., Ph. D., Office of Postgraduate Medical Education, Yokohama City University Hospital, 3-9 Fukuura, Kanazawa-ku, Yokohama, 236-0004, Japan Fax: +81-45-784-3546

inamorim@med.yokohama-cu.ac.jp

\section{ABSTRACT}

Background and study aims Lubiprostone has been reported to be an anti-constipation drug. The aim of the study was to investigate the usefulness of lubiprostone both for bowel preparation and as a propulsive agent in small bowel endoscopy.
Patients and methods This was a double-blind, placebocontrolled, 2-way crossover study of subjects who volunteered to undergo capsule endoscopy (CE). A total of 20 subjects ( 16 male and 4 female volunteers) were randomly assigned to receive a $24-\mu \mathrm{g}$ tablet of lubiprostone $120 \mathrm{~min}$ utes prior to capsule ingestion for CE (L regimen), or a placebo tablet 120 minutes prior to capsule ingestion for CE ( $P$ regimen). Main outcome was gastric transit time (GTT) and small-bowel transit time (SBTT). Secondary outcome was adequacy of small-bowel cleansing and the fluid score in the small bowel. The quality of the capsule endoscopic images and fluid in the small bowel were assessed on 5-point scale.

Results The capsule passed into the small bowel in all cases. Median GTT was $57.3(3-221)$ minutes for the $P$ regimen and $61.3(10-218)$ minutes for the $L$ regimen $(P=$ 0.836). Median SBTT was $245.0(164-353)$ minutes for the $P$ regimen and $228.05(116-502)$ minutes for the $L$ regimen $(P=0.501)$. The image quality score in the small bowel was $3.05 \pm 1.08$ for the $P$ regimen and $3.80 \pm 0.49$ for the $L$ regimen $(P<0.001)$. The fluid score in the small bowel was $2.04 \pm 1.58$ for the $P$ regimen and $2.72 \pm 1.43$ for the $L$ regimen $(P<0.001)$. There was a significant difference between the 2 regimens with regard to image quality. The fluid score was more plentiful for the $L$ regimen than for the $P$ regimen. There were no cases of capsule retention or serious adverse events in this study.

Conclusion Our study showed that use of lubiprostone prior to CE significantly improved visualization of the small bowel during $\mathrm{CE}$ as a result of inducing fluid secretion into the small bowel.

\section{Introduction}

Capsule endoscopy (CE) has been established as a convenient method for evaluating the small bowel. CE provides a higher diagnostic yield than barium contrast radiography of the small bowel or enteroscopy [1-4]. It is safe, painless, and well-tolerated [5]. Despite these advantages, the diagnostic yield of CE may be restricted by some limitations, including technical difficulties, inability of some patients to swallow the capsule, the relatively poor quality of the small-bowel images, and the frequent inability of this modality to allow complete assessment of the small bowel; in $20-30 \%$ of the cases, the capsule does not reach the cecum within the imaging period [6-8]. In addition, overall results differ among studies, with the reported percentage of cases of incomplete visualization of the mucosal surfaces due to bubbles, or luminal residue obscuring the view, especially in the distal small bowel, varying from $5 \%$ to $30 \%$ [9$12]$. 


\begin{tabular}{|c|c|c|c|}
\hline & $P$ regimen & $L$ regimen & $P$ value \\
\hline Subjects number & 10 & 10 & \\
\hline Age & $33.5(29-53)$ & $32.3(30-41)$ & 0.898 \\
\hline Sex (male / female) & $8 / 2$ & $8 / 2$ & - \\
\hline Height (cm) & $172.6(154-178)$ & $168.7(150-183)$ & 0.732 \\
\hline Weight (kg) & $66.5(49-88)$ & $65.1(46-92)$ & 0.485 \\
\hline Body-mass index $\left(\mathrm{kg} / \mathrm{m}^{2}\right)$ & $22.3(17.8-33.5)$ & $22.9(17.5-33.8)$ & 0.824 \\
\hline Drinking history & 3 & 5 & 0.275 \\
\hline Smoking history & 2 & 1 & 0.211 \\
\hline Abdominal surgery & 0 & 0 & - \\
\hline History of intestinal obstruction & 0 & 0 & - \\
\hline History of autoimune disorders & 0 & 0 & - \\
\hline
\end{tabular}

Several studies have examined the possibility of shortening transit time and improving bowel cleanness by using different medications for bowel preparation and prescribing different fasting periods $[13,14]$. Current bowel preparation protocols, although still not standardized, usually include clear liquids the day before, and restraint from drinking and eating within 8 to 10 hours before capsule ingestion. Comparisons of this type of preparation with gut lavage or oral sodium phosphate regimens have shown similar efficacy for all the regimens [15]. Therefore, the optimal preparation method for small-bowel CE has not yet been established.

Lubiprostone (Amitiza; ABBOTT JAPAN CO., LTD, Tokyo) selectively activates the type- 2 chloride channels in the apical membrane of the gastrointestinal epithelium, inducing net fluid secretion. It is currently approved by the US Food and Drug Administration for treatment of chronic idiopathic constipation, constipation-predominant irritable bowel syndrome in women and opioid-induced constipation in patients with chronic non-cancer pain. The proposed primary mechanism of action of lubiprostone in the gastrointestinal tract is increased chloride ion transport into the intestinal lumen by the drug caused by the opening of $\mathrm{ClC}-2$, which results in increased intestinal secretion and accelerated mass transit $[16,17]$. Transit time studies by Camilleri et al. [18] revealed that lubiprostone accelerated small bowel transit and colonic transit times. Prior to this report, some clinical studies by our group showed that lubiprostone decreased gastric and small-bowel transit time [19-20]. Meta-analysis showed differing results for gastric and small-bowel transit times and the reasons for that are not yet clear [21-22].

To solve this problem, we first examined the preliminary study to find the optimum intake time for lubiprostone before the CE [19], then we designed a double-blind, placebo-controlled 2-way crossover trial to investigate the usefulness of lubiprostone, both as a bowel preparation agent and as a propulsive agent for small bowel endoscopy.

\section{Patients and methods Trial design}

This was a double-blind, placebo-controlled, 2-way crossover study of subjects who volunteered to undergo CE. In all subjects, CE was performed with the PillCam SB2 CE system (Given Imaging Ltd), and the images were viewed with the Rapid 5 Reader. The subjects were randomly assigned to receive a 24- $\mu$ g tablet of lubiprostone 120 minutes prior to capsule ingestion for CE (L regimen), or a placebo tablet 120 minutes prior to capsule ingestion for $C E$ ( $P$ regimen). Subjects receiving either regimen were allowed to drink mineral water (maximum $1000 \mathrm{~mL}$ ) 2 hours after swallowing the capsule and to eat a light meal (balanced food: calorie mate ${ }^{\circledR} 800 \mathrm{kcal}$ ) after 4 hours. The subjects did not eat and drink anything else until the test was completed. After 8 hours, they returned to the endoscopy unit where the recorder was removed and the images were downloaded. Each of the test conditions were separated by a washout period of at least 7 days.

\section{Participants}

The study was performed between April 2014 and June 2014 at Yokohama City University School of Medicine. The subjects were 16 male volunteers and 4 female volunteers (average age: 32.9 years; age range: $29-53$ years). Baseline evaluations included a medical history, physical examination, and collection of demographic data ( $\vee$ Table 1 ). Exclusion criteria were a history of gastric or intestinal surgery, clinical or suspected abnormalities of gastric emptying, pregnancy or possible pregnancy, age $<18$ years, and a history of intake of medications during the previous week that could potentially affect the gastrointestinal motility. 
- Table 2 Transit time of the capsule endoscope.

\begin{tabular}{|l|l|l|l|}
\hline & P regimen & L regimen & P value \\
\hline Gastric transit time $(\mathrm{min})$ & 57.3 & 61.3 & $\mathbf{0}$ \\
\hline Small bowel transit time $(\mathrm{min})$ & $(3-221)$ & $\mathbf{( 1 0 - 2 1 8 )}$ & 0.970 \\
\hline Median (Minimum - maximum). $P$ values were calculated by the Wilcoxon signed rank test. & & 0.368 \\
\hline
\end{tabular}

\section{Intervention}

Subjects receiving either regimen were instructed to have nothing by mouth for at least 8 hours prior to capsule ingestion for CE. Lubiprostone or placebo was administered 120 minutes prior to capsule ingestion in accordance with the protocols described above. The PillCam Small Bowel CE system (Given Imaging, Yoqneam, Israel) with the PillCam SB2 capsule and Rapid 5 software platform were used for the study. All CE images were read by 2 investigators (M.I. and M. M.), both of whom were blinded to the group allocation status of the subjects. Small bowel examination was considered to be complete if the capsule had passed into the colon.

\section{Outcome}

The main outcome was gastric transit time (GTT) and smallbowel transit time (SBTT). The secondary outcome was adequacy of small-bowel cleansing and the fluid score in the small-bowel.

\section{Gastric and small-bowel transit times}

GTT was calculated from the time the capsule entered the stomach until it crossed the pylorus. SBTT was determined as the time from the first duodenal image until the capsule entered the colon, and could be calculated only in cases in which the capsule had reached the colon.

\section{Adequacy of bowel preparation}

Quality assessment of the capsule endoscopic images was made in accordance with the scale used by Aymer et al., with some modification [17, 23-25]. We used a 5-point scale (0-4) based on the percentage of the capsule images that were unimpaired by presence of debris or dark luminal fluid (4, $100-80 \%$; $3,80-60 \% ; 2,60-40 \% ; 1,40-20 \% ; 0,20-0 \%$ ). The average scores for 5 -minute segments of the video were assessed from capsule entry into the proximal duodenum ( $0 \%$ of the SBTT) and for every $10 \%$ of the SBTT thereafter, with the score for the final segment recorded in the terminal ileum ( $100 \%$ of the SBTT). We assessed the proximal $(0,10,20$, and $30 \%$ of the SBTT), middle $(40,50$, and 60 of the SBTT), distal $(70,80,90$, and $100 \%$ of the SBTT) and all $(0,10,20,30,40,50,60,70$, 80,90 and $100 \%$ of the SBTT) of the small bowel.

\section{Assessment of the fluid score in the small bowel}

We used a 5-point scale $(0-4)$ based on the percentage of the capsule endoscopic images that showed the fluid score (4, $100-80 \%$; 3, $80-60 \% ; 2,60-40 \% ; 1,40-20 \%$; $0,20-0 \%$ ). The average scores for 5 -minute segments of the video were assessed from capsule entry into the proximal duodenum ( $0 \%$ of the SBTT) and for every $10 \%$ of the SBTT thereafter, with the score for final segment recorded in the terminal ileum (100\% of the SBTT). We assessed the proximal $(0,10,20$, and $30 \%$ of the SBTT), middle $(40,50$, and 60 of the SBTT), distal $(70,80,90$, and $100 \%$ of the SBTT) and all $(0,10,20,30,40,50,60,70$, 80,90 and $100 \%$ of the SBTT) of the small bowel.

\section{Ethics}

The study was conducted in accordance with the Declaration of Helsinki. The study protocol was approved by the Ethics Committee of Yokohama City University Hospital. All the patients provided their written informed consent.

\section{Statistical analysis}

Statistical evaluation was performed using the $t$ test and Wilcoxon's signed rank test. The level of significance was set at $P<0.05$. All statistical analyses were performed with EZR (Saitama Medical Center, Jichi Medical University), which is a graphical user interface for R (The R Foundation for Statistical Computing). In other words, it is a modified version of the R commander designed to add statistical functions frequently used in biostatistics $[26,27]$.

\section{Results}

All 20 enrolled subjects completed the study. The subjects were divided into 2 groups ( $P$ regimen, $L$ regimen) and their characteristics are shown in $>$ Table 1 . No significant difference was seen between the $2 \mathrm{~s}$ groups. No adverse events (AEs) occurred during the study. All subjects received placebo and lubiprostone in a cross-over setup and swallowed the SB2 capsule, with the endoscopic images recorded for 8 hours. There were no cases of capsule retention after the study was completed. The capsule passed into the small bowel in all cases.

Median GTT observed was $57.3(3-221)$ minutes for the $P$ regimen and $61.3(10-218)$ minutes for the $\mathrm{L}$ regimen $(P=$ 0.836). Median SBTT observed was 245.0 (164-353) minutes for the $P$ regimen and 228.05 (116-502) minutes for the $L$ regimen $(P=0.501)$. The data are summarized in $>$ Table 2 .

The image quality scores were $3.58 \pm 0.67,3.35 \pm 0.8,2.1 \pm$ 1.15 and $3.05 \pm 1.08$ for the $P$ regimen, $3.95 \pm 0.29,3.85 \pm 0.39$ $3.58 \pm 0.67$ and $3.80 \pm 0.49$ for the $L$ regimen in proximal $(P<$ $0.001)$, middle $(P<0.001)$, distal $(P<0.001)$ and all $(P<0.001)$ of the small bowel. Fluid scores were $2.73 \pm 1.35,2.06 \pm 1.60$, $1.30 \pm 1.45$ and $2.04 \pm 1.58$ for the $P$ regimen, $3.57 \pm 0.87$, $2.71 \pm 1.40,1.87 \pm 1.44$ and $2.72 \pm 1.43$ for the $L$ regimen in 
- Table 3 Scores for image quality and fluid.

\begin{tabular}{|l|l|l|l|}
\hline & P regimen & L regimen & P value \\
\hline Image quality score & & & $<0.001$ \\
\hline - Proximal & $3.58 \pm 0.67$ & $3.95 \pm 0.29$ & $<0.001$ \\
\hline - Middle & $3.35 \pm 0.8$ & $3.85 \pm 0.39$ & $<0.001$ \\
\hline - Distal & $2.1 \pm 1.15$ & $3.58 \pm 0.67$ & $<0.001$ \\
\hline - All & $3.05 \pm 1.08$ & $3.80 \pm 0.49$ & $<0.001$ \\
\hline Fluid score & & & $<0.001$ \\
\hline - Proximal & $2.73 \pm 1.35$ & $3.57 \pm 0.87$ & $<0.001$ \\
\hline - Middle & $2.06 \pm 1.60$ & $2.71 \pm 1.40$ & $<$ \\
\hline - Distal & $1.30 \pm 1.45$ & $1.87 \pm 1.44$ & $<0.001$ \\
\hline - All & $2.04 \pm 1.58$ & $2.72 \pm 1.43$ & \\
\hline Average \pm standard deviation (SD). P values were calculated by the Wilcoxon signed rank test. & & \\
\hline
\end{tabular}

the proximal $(P<0.001)$, middle $(P<0.001)$, distal $(P<0.001)$ and all $(P<0.001)$ of the small bowel. The data are summarized in $>$ Table 3.

Image quality became worse as the capsule progressed more distally. The views were impaired by bile, residue, and bubble artifacts. On the other hand, image quality scores for the $L$ regimen tended to be better than those for the $P$ regimen for all small bowel segments. There was a significant difference between the 2 regimens with regard to image quality $(P<$ 0.001) ( Fig. 1). On the other hand, the amount of fluid was more plentiful with the $L$ regimen than with the $P$ regimen for all small bowel segments ( $>$ Fig. 2). There were no cases of capsule retention or serious AEs in this study.

\section{Discussion}

This study was designed to evaluate the effect of lubiprostone on the capsule transit time through the gastrointestinal lumen and its effectiveness as a bowel preparation agent for improving the quality of capsule imaging of the small bowel. Lubiprostone improved the imaging quality of the small bowel as compared to placebo. Lubiprostone did not improve the SBTT and did not delay the GTT.

Lubiprostone is approved to treat chronic constipation, constipation-predominant irritable bowel syndrome and opioid-induced constipation in non-cancer-related chronic pain. The proposed primary mechanism of action is an increase in chloride ion transport into the intestinal lumen caused by opening up by the drug of $\mathrm{ClC}-2$ channels, leading to increased intestinal secretion and accelerated mass transit. GTT following administration of lubiprostone was similar to that after administration of placebo. Our findings differ from those in the study reported by Camilleri et al. [18], who reported finding evidence of delayed gastric emptying following administration of lubiprostone. The main side effect of lubiprostone was nausea, possibly related to delayed gastric emptying [18-22]. In Phase II trials, nausea was reported in as many as $33 \%$ of patients receiving 48

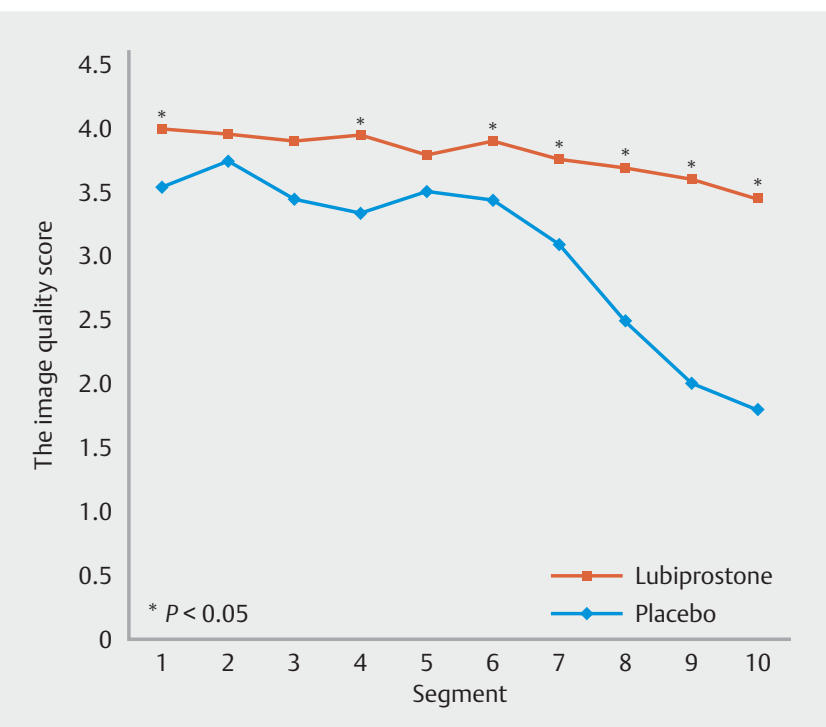

- Fig. 1 Image quality score. Visibility of the mucosal surface was assessed as the percentage of visualized bowel surface area: 1 , $<25 \%$; 2, 25-49\%; 3, 50-74\%; 4, 75-89\%; and 5, $\geq 90 \%$.

$\mu \mathrm{g}$ of lubiprostone daily [28]. Nausea was the most common side effect of lubiprostone, reported in up to $31 \%$ of patients in 1 study [29]. Several possible explanations for nausea have been suggested, including delayed gastric emptying, small intestinal distention secondary to increased gastric secretion, change in gastrointestinal sensation, and/or additional actions of lubiprostone on gastrointestinal motility [18-22]. However, the precise mechanism of nausea associated with lubiprostone remains unclear.

SBTT following administration of lubiprostone was similar to that after administration of placebo. Our findings differ from those of the study by Camilleri et al. [18] in 2006. They found a significant acceleration of SBTT along with accelerated colonic 


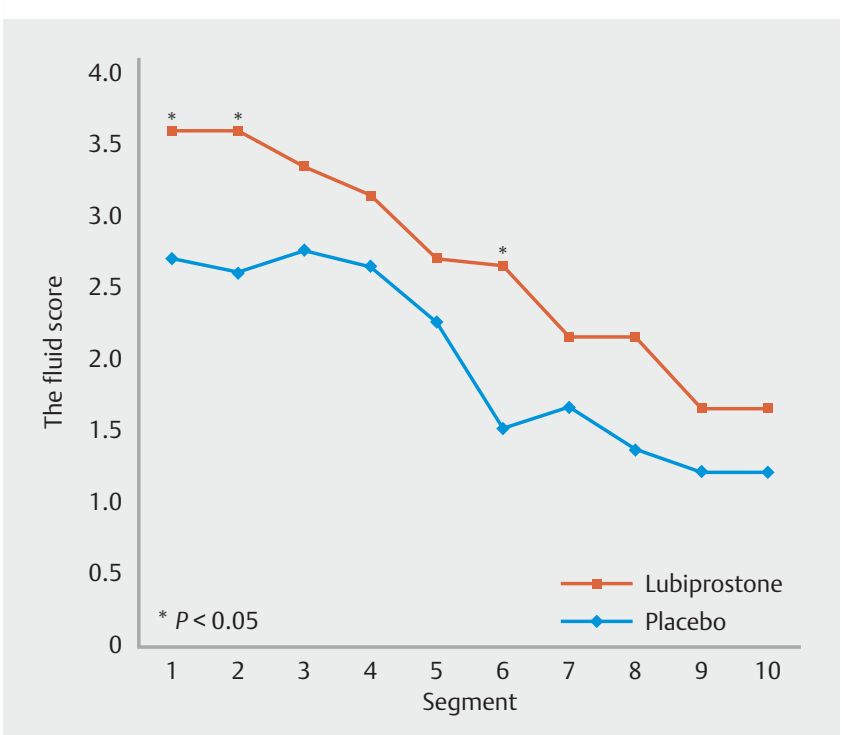

- Fig. 2 Fluid score. Fluid of endoscopic image was assessed as the percentage of the fluid area: $1,<25 \% ; 2,25-49 \% ; 3,50-74 \% ; 4$, $75-89 \%$; and $5, \geq 90 \%$.

transit time. However, Hooks et al. [20] showed that lubiprostone did not decrease SBTT.

Our preliminary study [19] also suggested that lubiprostone has been shown to accelerate overall colonic transit without significantly changing the rate of emptying of the ascending colon [17]. With the proximal colon likely reabsorbing the increased fluid load from the small intestine, it is postulated that a primary motor effect on the colon beyond the ascending portion may be responsible for this effect [30]. Similarly, presence of a possible direct smooth muscle effect of lubiprostone on the rest of the gastrointestinal tract has also been suggested.

Lubiprostone selectively activates type 2 chloride channels in the apical membrane of the gastrointestinal epithelium, resulting in net fluid secretion [20]. It has been approved by the FDA for treatment of chronic idiopathic constipation and constipation-predominant IBS. Recent studies revealed that it accelerates small-bowel transit as well as colonic transit time [21].

The use of polyethylene glycol (PEG) before capsule administration has yielded mixed results on intestinal propulsion and bowel preparation efficacy. There are 2 reports of studies in which PEG was given after capsule administration for $C E$, both of which showed promising results. Fireman et al. [31] reported retrospectively that patients who received $1.5 \mathrm{~L}$ of PEG 12 hours before capsule ingestion and $1.5 \mathrm{~L}$ of PEG 1 hour after capsule ingestion showed significant shortening of transit time through the stomach and small bowel as compared to patients who were bowel-prepared with sodium phosphate and those with no colon preparation. Endo et al. [20] used a standard liquid diet and nothing by mouth for initial preparation and gave patients $500 \mathrm{~mL}$ of PEG 30 minutes after capsule ingestion. Administration of PEG post-capsule ingestion resulted in an increased rate of cecal entry of the capsule and improved distal small bowel imaging.
It has been reported that use of prokinetics such as metoclopramide [23,25], erythromycin [14] and mosapride [32] may decrease randomness in the rate of gastric emptying and reduce SBTT. Selby [23] reported that administration of oral metoclopramide before capsule administration reduced GTT with no effect on SBTT, but still had a positive effect by increasing the percentage of capsules reaching the cecum. Metoclopramide, with the addition of senna and citrate of magnesia for bowel preparation, has also been shown to improve both GTT and SBTT. Metoclopramide has several actions that may account for its favorable influence on capsule transit time. Its main effect is in the proximal gastrointestinal tract. It improves gastric tone and peristalsis, relaxes the pyloric sphincter, and improves antroduodenal coordination [33] by a combination of its cholinergic and antidopaminergic effects [34].

The current study has some limitations. First, the number of study subjects was small, although statistically significant differences in image quality score and fluid score in the small bowel were observed between the 2 study groups. Second, our results may be biased, because only a few female subjects were enrolled in this study. Some studies have reported an influence of gender on gastrointestinal motility, with transit time in females tending to be longer than that in males [35-39].

\section{Conclusion}

Our study showed that use of lubiprostone prior to CE significantly improved visualization of the small bowel during CE as a result of induction of fluid secretion into the small bowel.

Author's disclosure of potential Conflict of Interest (COI). Masahiko

\section{Acknowledgements}

The study was supported by a research grant from Abbott Japan Co., LTD.

\section{Competing interests}

Dr. Inamori receives research funding from Abbott Japan Co., LTD.

\section{References}

[1] Lewis BS, Swain P. Capsule endoscopy in the evaluation of patients with suspected small intestinal bleeding: results of a pilot study. Gastrointest Endosc 2002; 56: 349-353

[2] Eliakim R, Fischer D, Suissa A et al. Wireless capsule video endoscopy is a superior diagnostic tool in comparison to barium follow through and computerized tomography in patients with suspected Crohn's disease. Eur J Gastroenterol Hepatol 2003; 15: 363-367

[3] Hartmann D, Schilling D, Bolz G et al. Capsule endoscopy versus push enteroscopy in patients with occult gastrointestinal bleeding. Z Gastroenterol 2003; 41: 377-382

[4] Costamagna G, Shah SK, Riccioni ME et al. A prospective trial comparing small bowel radiographs and video capsule endoscopy for 
suspected small bowel disease. Gastroenterology 2002; 123: 999 1005

[5] Appleyard M, Glukhovsky A, Swain P. Wireless-capsule diagnostic endoscopy for recurrent small bowel bleeding. N Engl J Med 2001; 344: $232-233$

[6] Rondonotti E, Herrerias JM, Pennazio M et al. Complications, limitations, and failures of capsule endoscopy: a review of 733 cases. Gastrointest Endosc 2005; 62: 712 - 716

[7] Carey EJ, Leighton JA, Heigh RI et al. A single-center experience of 260 consecutive patients undergoing capsule endoscopy for obscure gastrointestinal bleeding. Am J Gastroenterol 2007; 102: 89-95

[8] Tatar EL, Shen EH, Palance AL et al. Clinical utility of wireless capsule endoscopy: experience with 200 cases. J Clin Gastroenterol 2006; 40: $140-144$

[9] Niv Y, Niv G. Capsule endoscopy: role of bowel preparation in successful visualization. Scand J Gastroenterol 2004; 39: 1005-1009

[10] Dai N, Gubler C, Hengstler P et al. Improved capsule endoscopy after bowel preparation. Gastrointest Endosc 2005; 61: 28 - 31

[11] Ben-Soussan E, Savoye G, Antonietti M et al. Is a 2-liter PEG preparation useful before capsule endoscopy? J Clin Gastroenterol 2005; 39 : $381-384$

[12] Van Tuyl SA, den Ouden H, Stolk MF et al. Optimal preparation for video capsule endoscopy: a prospective, randomized, single-blind study. Endoscopy 2007; 39: 1037 - 1040

[13] Viazis N, Sgouros S, Papaxoinis K et al. Bowel preparation increases the diagnostic yield of capsule endoscopy: a prospective, randomized, controlled study. Gastrointest Endosc 2004; 60: 534- 538

[14] Leung WK, Chan FK, Fung SS et al. Effect of oral erythromycin on gastric and small bowel transit time of capsule endoscopy. World J Gastroenterol 2005; 11: 4865 - 4868

[15] Kalantzis C, Trantafyllou K, Papadopoulus AA et al. Effect of three bowel preparations on video-capsule endoscopy gastric and smallbowel transit time and completeness of the examination. Scand J Gastroenterol 2007; 42: 1120 - 1126

[16] Cuppoletti ], Malinowska DH, Tewari KP et al. SPI-0211 activates T84 cell chloride transport and recombinant human CIC-2 chloride currents. Am J Physiol 2004; 287: C1173 - C1183

[17] Postgate A, Tekkis P, Patterson N et al. Are bowel purgatives and prokinetics useful for small bowel capsule endoscopy? A prospective randomized controlled study Gastrointest Endosc 2009; 69: 1120 1128

[18] Camilleri M, Bharucha AE, Ueno R et al. Effect of a selective chloride channel activator, lubiprostone, on gastrointestinal transit, gastric sensory, and motor functions in healthy volunteers. Am J Physiol Gastrointest Liver Physiol 2006; 290: G942 - G947

[19] Matsuura M, lida H, Nonaka T et al. Lubiprostone decreases smallbowel transit time and improves visualization of the small bowel in capsule endoscopy: a double- blind, placebo-controlled 3-way crossover study. Gastroenterology Research and Practice 2014; 146: S358

[20] Hooks SB3rd, Rutland T], Di Palma JA et al. Lubiprostone neither decreases gastric and small-bowel transit time nor improves visualization of small bowel for capsule endoscopy: a double-blind, placebocontrolled study. Gastrointest Endosc 2009; 70: 942 - 946

[21] Koulaouzidis A, Giannakou A, Yung DE et al. Do prokinetics influence the completion rate in smallbowel capsule endoscopy? A systematic review and meta-analysis Curr Med Res Opin 2013; 29: 1171 - 1185
[22] Giannakou A, Dabos KJ, Koulaouzidis A et al. Lubiprostone in smallbowel capsule endoscopy: meta-analyzing the data. Gastrointest Endosc 2015; 81: 1047 - 1048

[23] Selby W. Complete small bowel transit in patients undergoing capsule endoscopy: determining factors and improvement with metoclopramide. Gastrointest Endosc 2005; 61: 80 - 85

[24] Endo $\mathrm{H}$, Kondo $\mathrm{Y}$, Inamori $\mathrm{M}$ et al. Ingesting $500 \mathrm{ml}$ of polyethylene glycol solution during capsule endoscopy improves the image quality and completion rate to the cecum. Dig Dis Sci 2008; 53: $3201-3205$

[25] Hosono K, Endo H, Sakai E et al. Optimal approach for small bowel capsule endoscopy using polyethylene glycol and metoclopramide with the assistance of a real-time viewer. Digestion 2011; 84: 119 125

[26] Kanda Y. Investigation of the freely-available easy-to-use software "EZR" (Easy R) for medical statistics. Bone Marrow Transplant 2013; 48: $452-458$

[27] Kikkawa N, Inamori M, Inoue S et al. Comparative study of the QUEST questionnaire and GerdQ questionnaire for Japanese students. Hepatogastroenterology 2014; 61: 1605-1610

[28] Johanson JF, Ueno R. Lubiprostone, a locally acting chloride channel activator, in adult patients with chronic constipation: a double-blind, placebo-controlled, dose-ranging study to evaluate efficacy and safety. Aliment Pharmacol Ther 2007; 25: 1351 -1361

[29] Hussar DA. New drugs: lubiprostone, ranolazine, and anidulafungin. J Am Pharm Assoc 2006; 46: 411 - 414

[30] Sweetser S, Busciglio IA, Camilleri M et al. Effect of a chloride channe activator, lubiprostone, on colonic sensory and motor functions in healthy subjects. Am J Physiol Gastrointest Liver Physiol 2009; 296 : G295-G301

[31] Fireman Z, Kopelman Y, Fish L et al. Effect of oral purgatives on gastric and small bowel transit time in capsule endoscopy. Isr Med Assoc J 2004; 6: $521-523$

[32] Wei W, Ge ZZ, Lu H et al. Effect of mosapride on gastrointestinal transit time and diagnostic yield of capsule endoscopy. J Gastroenterol Hepatol 2007; 22: $1605-1608$

[33] Rabine JC, Barnett JL. Management of the patient with gastroparesis. J Clin Gastroenterol 2001; 32: 11 - 18

[34] Ramsbottom N, Hunt JN. Studies of the effect of metoclopramide and apomorphine on gastric emptying and secretion in man. Gut 1970; 11: $989-993$

[35] Ristvedt SL, McFarland EG, Weinstock LB et al. Patient preferences for CT colonography, conventional colonoscopy and bowel preparation. Am J Gastroenterol 2003; 98: 578-575

[36] Knight LC, Parkman HP, Brown KL et al. Delayed gastric emptying and decreased antral contractility in normal premenopausal women compared with men. Am J Gastroenterol 1997; 92: 968 -975

[37] Parkman HP, Harris AD, Miller MA et al. Influence of age, gender, and menstrual cycle on the normal electrogastrogram. Am J Gastroenterol 1996; 91: $127-133$

[38] Wedmann B, Schmidt G, Wegener M et al. Effects of age and gender on fat-induced gallbladder contraction and gastric emptying of a caloric liquid meal: a sonographic study. Am J Gastroenterol 1991; 86: $1765-1770$

[39] Huston WR, Roehrkasse RL, Wald A et al. Influence of gender and menopause on gastric emptying and motility. Gastroenterology 1989; 96: $11-17$ 